\title{
DENGUE FEVER WITH PULMONARY ALVEOLAR HEMORRHAGE - COMMON INFECTION WITH UNCOMMON ASSOCIATION - RARE CASE REPORT
}

\author{
B SAROJ KUMAR PRUSTY1 ${ }^{1}$ MAJED ABDUL BASIT MOMIN²
}

\begin{abstract}
:
Dengue fever $(D F)$ is major health hazard around the world in recent decades especially in India. Flu like illness to polyserositis among common presentation of DF. Dengue fever presenting as pulmonary hemorrhage is rare. Here we present a case of 16 -year young male patient presented with fever and shortness of breath, initially diagnosed as dengue fever by serological examination. CT chest findings mimiking as pneumonia, however further fall in hemoglobin and progressive dysnoea, bronchoscopy and bronchial wash cytology done which reveal association of pulmonary hemorrhage. This case report alerted clinicians about rare presentation and high index of suspicion of pulmonary hemorrhage in DF with dysnoea for early diagnosis and to avoid its fatal outcome.
\end{abstract}

Key-words: Dengue fever ; Bronchoalveolar lavage ; Pulmonary hemorrhage

Received: 08 October 2018

Accepted: 05 November 2018

DOI: https://doi.org/10.3329/bjmed.v30i1.39922

\section{Introduction:}

$\mathrm{DF}$ is mosquito born disease, and its severe forms namely, dengue hemorrhagic fever (DHF) and dengue shock syndrome (DCS) transmitted by bite of mosquito infected with one of the four dengue viruse serotypes. Dengue is widespread throughout developed and developing countries, with local variations in risk influenced by rainfall, temperature and rapid urbanization. Dengue disease has wide spectrum of clinical signs and symptoms ranging from asymptomatic infection to severe and fatal manifestation. ${ }^{1}$ So far, dengue with pulmonary alveolar hemorrhage rarely been described in literature. Radiological imaging, chest radiography and $\mathrm{HRCT}$ findings in pulmonary manifestations are supportive but not diagnostic. ${ }^{2}$ However bronchoscopy with bronchial lavage cytology with special perls prussian blue stain play crucial role to diagnose pulmonary hemorrhage. ${ }^{3}$

\section{Case Report :}

A 16-year young boy presented with shortness of breath for a day and history of high grade fever associated with chills and rigors for five days. Patient initially evaluated outside hospital with hemoglobin $17 \mathrm{gm} \%$, white blood cells $4500 /$ cumm and platelet count was 1.2 lakhs/cumm. On general examination patient was conscious, febrile (102 F), tachycardia (126 beats/min), respiratory rate was $28 / \mathrm{min}$ and blood pressure $100 / 7 \mathrm{mmHg}$. Systemic examination normal except respiratory system with decreased air entry in left infraxillary area and bilateral crepitations.

Laboratory hematological results reveal Hemoglobin $13.1 \mathrm{gm} \%$, RBCs count 4.33 millions $/$ cumm, hematocrit $40 \%$, White blood cells count $8000 /$ cumm,platelets 95000/cumm and normal differential on morphology. ESR was $50 \mathrm{~mm} / \mathrm{hr}$. Coagulation study reveal elevated prothrombin time $18 \mathrm{sec}$ (control12.9sec) and INR 1.5. APTT within normal limit. Biochemical parameters including LFT, Renal function test were in normal limits. Serological dengue Ig M positive by ELISA. Malarial antigen, Typhi IgM and scrub typhus were negative.

Chest $X$ ray which was done outside was normal, however in view of progressive dysnoea inspite of supportive measures and considering previous hemoglobin value possibility of pulmonary hemorrhage suspected and underwent CT scan chest and bronchoscopy. CT scan chest show multifocal areas of patchy consolidation and groungd glass hazziness in left lung field and right lower lobe (Figure 1 \&2) suggestive of infective etiology. Bronchoscopic examination revealed erythematous (Figure 3a) mucosa more in left lung and subsequent bronchial wash cytology show many pigment laden macrophages (Figure 3b,c,d), which was confirmed by special stain for hemosiderin (Perls stain). Bronchial wash gene

1. Consultant Intensivist MD FICCM

2. Consultant pathologist

Address of Correspondence: Dr.B Saroj Kumar Prusty, Consultant Intensivist MD FICCM

Bangladesh J Medicine 2019; 30 : 38-40 
BJM Vol. 30 No. 1 Dengue Fever with pulmonary alveolar hemorrhage - Common infection with uncommon association
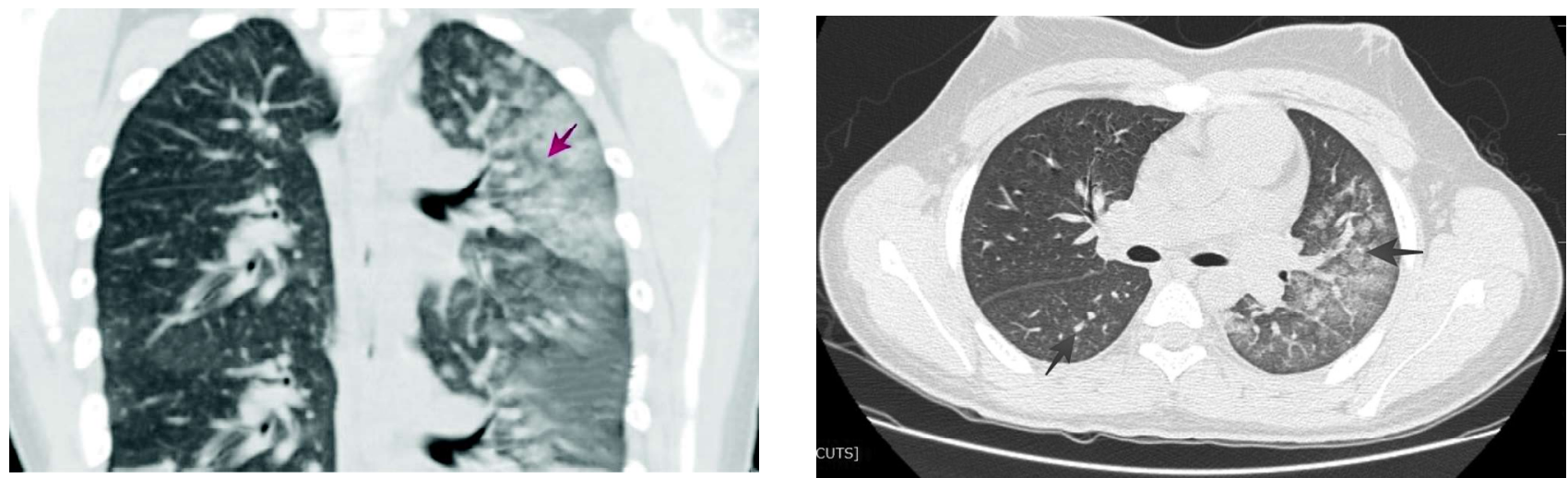

Fig.- 1 \& 2 : Coronal section \& Axial HRCT: multifocal areas of patchy consolidation and groungd glass hazziness in left lung field and right lower lobe
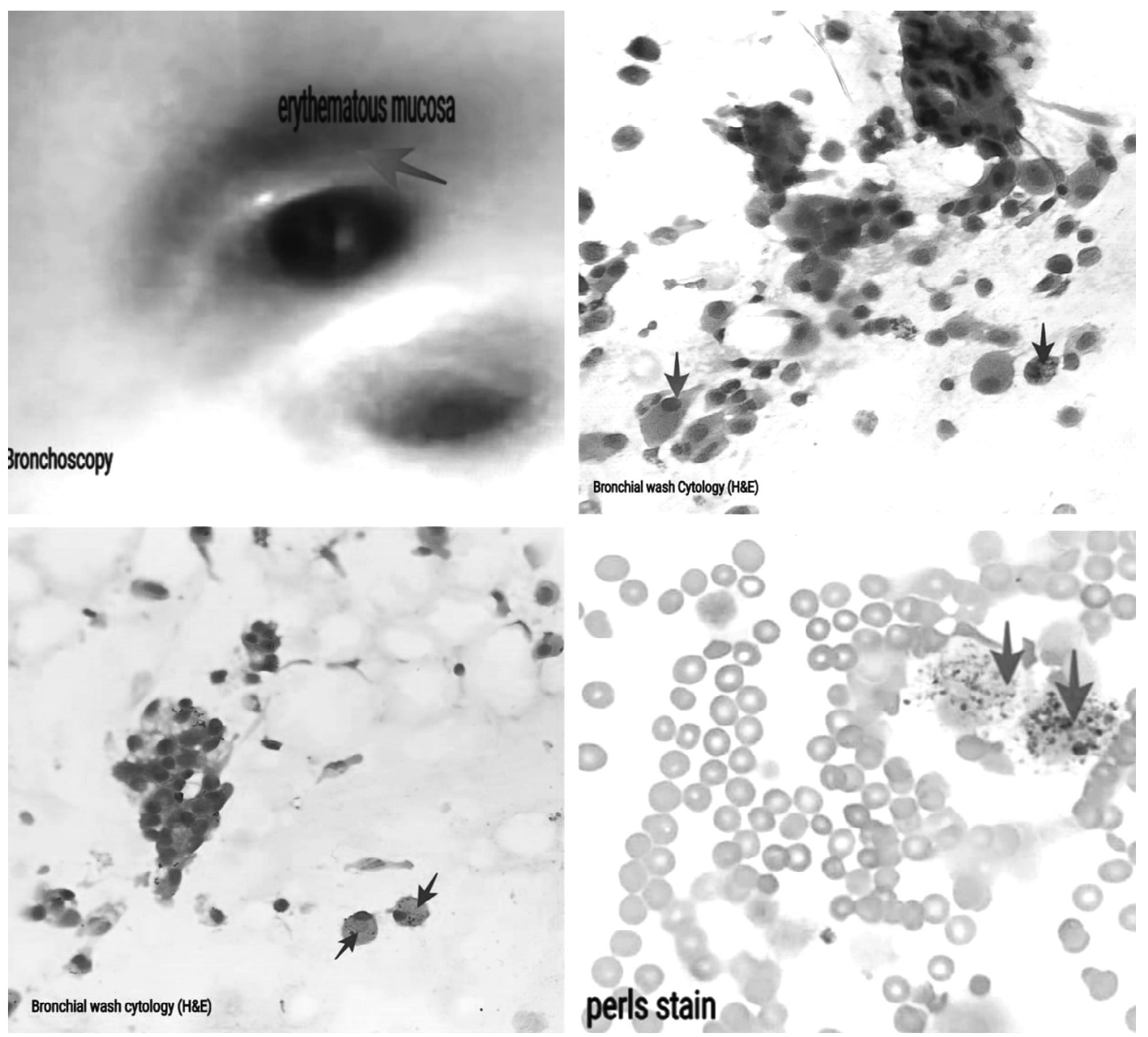

Fig.-3: (a) Bronchoscopy show erythematous mucosa with no active oozing of blood (b\&c) BAL cytology show many macrophages with no unflammatory cells (3d) Perls stain show hemosiderin laden macrophages with surrounding RBCs. 
Xpert for Myocbacterium tuberculosis came negative. Patient treated with supportive measures and improved symptomatically and with increased platelet count.

\section{DISCUSSION:}

The prevalence of dengue infection increased due to failure to control aedes population, uncontrolled urbanization, population growth,lack of vaccine and climate change. DF characterized by acute onset of high grade fever, 3 to 14 days after the bite of infected mosquitoes associated with reterorbital pain, myalgia, arthralgia and rash.

As per WHO criteria for DHF includes history of fever lasting for 2-7 days, thrombocytopenia(less than one lakh/ Cu/mm, any hemorrhagic manifestation and evidence of increased vascular permeability. Hemorrhagic manifestation are mild and includes positive tourniquet test, petechiae, ecchymosis, epistaxis and gum bleeding. More serious type of bleed includes per vaginal bleed, hematemesis, malena, pulmonary hemorrhage and intracranial hemorrhage. Evidence of vascular permeability consist of increased hematocrit $>20 \%$ or decline in hematocrit $>20 \%$, presence of pleural effusion to ascites by radiography and hypoprotenemia or hypoalbuminemia as determined by laboratory test. ${ }^{4}$ In present case patient had fever, thrombocytopenia and Dengue IgM positive by ELISA. Common course in DHF initially had fever lasting for two to seven days followed by phase of defervescence patient had thrombo-cytopenia, cutaneous lesions like petechiae and ecchymosis and rearely hemorrhagic manifestation in the form of epistaxis,bleeding gums, pulmonary hemorrhages, gastrointestinal hemorrhages and intracranial hemorrhages. ${ }^{5}$

Pulmonary manifestation in dengue infections includes pleural effusion, pneumonitis , hemoptysis, pulmonary hemorrhages and adult respiratory distress syndrome. The pathogenesis of pulmonary hemorrhage in dengue infection is not specific however possible etiology considered to be mulfactorial like low platelets, coagulation cascade abnormality, platelet dysfunction, vascular defect and disseminated intrvascular coagulation. Plasma leakage is secondary to increased vascular permeability possibly cytokine mediated. ${ }^{6}$ Classic triad seen in pulmonary hemorrhage includes hemoptysis ,rapid fall in hemoglobin over 24$48 \mathrm{hrs}$ and new interstitial infiltrate. ${ }^{7}$ In our case progressive dysnoea and rapid fall of hemoglobin make suspicion for pulmonary hemorrhage.

If alveolar hemorrhage suspected, early bronchoscopic gross finding and bronchoalveolar lavage sampling with red blood cell count and presence of hemosiderin laden macrophages diagnostic of alveolar hemorrhage and also to rule out trauma related $\mathrm{RBCs}$ and infection. Hemosiderin is a product of hemoglobin breakdown and appears at least 48 hours of bleeding and highlighted by special Perls stain. Beside supportive treatment in the form of critical monitoring of fluid balance and hydration ,there is no specific treatment. ${ }^{8}$ However bleeding manifestation with correction of coagulopathy require transfusion support.

\section{Conclusion:}

To conclude, though dengue infection are common, its association with pulmonary alveolar hemorrhage is rare finding. Clinicians should suspect pulmonary hemorrhage in case of progressive dysnoea and fall in hemoglobin. Bronchoscopy and brochoalveolar lavage with special Perls stain, play promising role to diagnose and manage this rare association, as imaging study mimics pneumonia like findings.

\section{References:}

1. Dengue bulletin; World Health Organization, Volume 39 december 2016

2. Edson Marchiori, José Luiz N Ferreira, -Pulmonary hemorrhage syndrome associated with dengue fever, High-resolution computed tomography findings Orphanet Journal of Rare Diseases 2009, 4:8 .https:/ /doi.org/10.1186/1750-1172-4-8

3. Capelozzi VL, Faludi EP, Balthazar AB, et al. Bronchoalveolar lavage improves diagnostic accuracy in patients with diffuse lung disease. Diagn Cytopathol 2013; 41:1. https://doi.org/10.1002/dc.21743.PMid: 21674814

4. Dengue Haemorrhagic Fever: Diagnosis, Treatment, Prevention and Control. Geneva, Switzerland, World Health Organization, 1997; ed 2, pp1-11.

5. S.K. Sharma, B.S. Gupta, G. Devpura, Pulmonary haemorrhage syndrome associated with dengue haemorrhagic fever, Journal of the Association of Physicians of India 55 (2007) 729-730. PMid:18173028

6. C. C. Michel and F. E. Curry, "Microvascular permeability," Physiological Reviews, vol. 79, no. 3, pp. 703-761, 1999..https://doi.org/10.1152/ physrev.1999.79.3.703.PMid:10390517

7. S. Santos-Ocampo, B. F. Mandell, and B. J. Fessler, "Alveolar hemorrhage : presentation and management," Chest, vol. 118, no. 4, pp. 1083-1090, 2000. https:// doi.org/10.1378/chest.118.4.1083.PMid:11035681

8. Lum LC, Abdel-Latif Mel-A, Goh AY, Chan PW, Lam SK (2003) Preventive transfusion in Dengue shock syndrome-is it necessary? J Pediatrics 143: 682-684. https://doi.org/ 10.1067/S0022-3476(03)00503-1 